\title{
Engineering a DNAzyme-based Operon System for the Production of DNA Nanoscaffolds in Living Bacteria
}

Dan M. Alon ${ }^{\dagger}$, Cristopher A. Voigt ${ }^{\ddagger}$ and Johann Elbaz ${ }^{\star \dagger}$

$\dagger \quad$ Mr. Dan Mark Alon, Dr. Johann Elbaz.

School of Molecular Cell Biology \& Biotechnology, Faculty of Life

Science

Tel Aviv University

Tel Aviv 69978, Israel.

joelbaz@tauex.tau.ac.il

$\ddagger \quad$ Prof. Christopher A. Voigt

Department of Biological Engineering, Massachusetts Institute of

Technology

Synthetic Biology Center

500 Technology Square

NE47-140

Cambridge, MA 02139 


\section{Table of Contents}

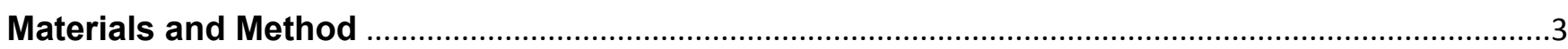

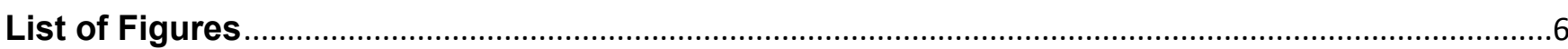

Figure S1. r_oligo Plasmid maps used for all experiments............................................................6

Figure S2. HIV reverse transcriptase Initiator plasmid map. ....................................................................7

Figure S4. Detailed scheme of the 4-part crossover motif and sequence color-coding ........................8

Figure S5. Expanded schematics and cytometry data corresponding to Figure 3a-c. ........................9

Figure S6. Expanded schematics and cytometry data corresponding to Figure 3b...........................10

Figure S7. Expanded schematics and cytometry data corresponding to Figure $3 \mathrm{~d}$............................11

Figure S8. Confocal microscopy images of the DNAzyme based operon system fluorescence in vivo

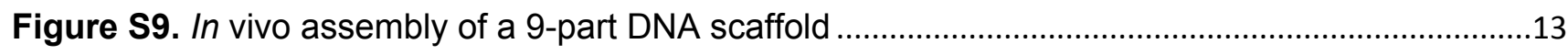

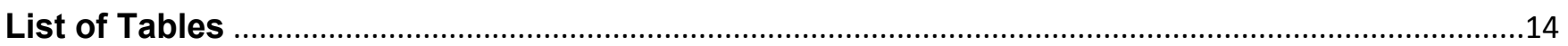

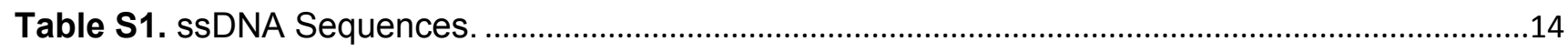

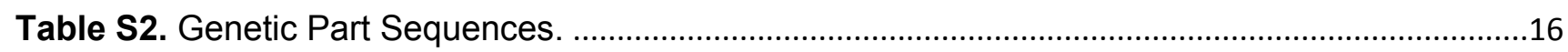

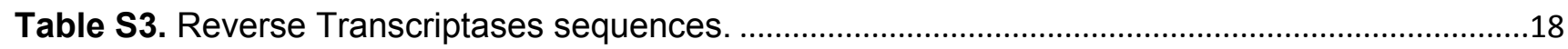

Table S4. Oligo sequences used during the short ssDNA sequencing assay. ....................................20

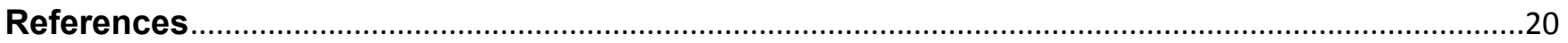




\section{Materials and Method}

\section{Strains and media}

Escherichia coli DH10 $\beta$ (MC1061 F - endA1 recA1 galE15 galK16 nupG rpsL $\Delta$ lacX74 क0lacZ $\Delta$ M15 araD139 $\Delta$ (ara,leu) mcrA $\Delta$ (mrr - hsdRMS - mcrBC) $\lambda$ - ) was used for all manipulations and assays. Cells were grown in BD difco LB Miller Broth (10 g/l tryptone, $5 \mathrm{~g} / \mathrm{l}$ yeast extract, $10 \mathrm{~g} / \mathrm{l} \mathrm{NaCl}$, Fisher Scientific). Ampicillin (100 $\mu \mathrm{g}$ per $\mu \mathrm{l}$, Affymetrix cat. \#11259-5), kanamycin (50 $\mathrm{g}$ per $\mu \mathrm{l}$, Gold Bio cat. \#K-120-5) and Spectinomycin (100 $\mu \mathrm{g}$ per $\mu \mathrm{l}$, MP Biomedicals cat. \#021 5899305) were used where appropriate. Isopropyl $\beta$-D-1-thiogalactopyranoside (IPTG, Roche cat. \#10 745740 001) or L-arabinose (L-ara, USB Corporation \# 532837 0) were used as inducers.

\section{DNA constructs}

All DNA sequences are provided in Tables S1 and S2. Key constructs will be made available via Addgene. The HIVRT p66 domain was codon optimized for E. coli and synthesized (Geneart). The p51 domain was obtained by PCR as a truncated version of this gene. The murine leukemia reverse transcriptase gene is not codon optimized. The HTBS part was ordered as a Gblock (IDT). The assembly of r_oligo genes into different versions of the r_oligo plasmids was performed using the Golden Gate Method ${ }^{1}$ or Gibson Assembly Method ${ }^{2}$. Plasmid maps are provided in Figures $\mathrm{S} 1,2,3$.

\section{In vivo ssDNA production and purification}

Cells were inoculated in $1 \mathrm{~L}$ of Terrific Broth containing the appropriate plasmids and incubated at $37^{\circ} \mathrm{C}$ at 250 r.p.m for $24 \mathrm{~h}$. After incubation, $50 \mathrm{~mL}$ of the resulting solution was purified using TRIzol Reagent protocol (Life

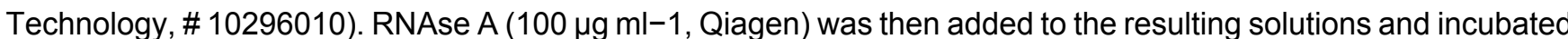
at $37^{\circ} \mathrm{C}$ for $2 \mathrm{~h}$. After RNA degradation, the solutions were run within PAGE (see below). This protocol has been used for experiments shown in Fig $2 b$.

\section{PAGE purification}

The excised gel slice was incubated in 400ul RNA Recovery Buffer (ZYMO Research Corp., R1070-1-10) at 65 $\mathrm{C}$ for $15 \mathrm{~min}$. The resulting solution was then placed into a Zymo-Spin IV Column (ZYMO Research Corp., C100750 ) and centrifuged at $9,391 \mathrm{~g}$ for $30 \mathrm{~s}$. The flow-through was then transferred into a tube including a $5 X$ volume of buffer PB (Qiagen) and $700 \mu \mathrm{l}$ of the resulting solution is transferred into QIAquick Spin Columns (Qiagen, Mat. No. 1018215) and centrifuged at 9,391g for $30 \mathrm{~s}$. This was repeated until all of the solution passed through the

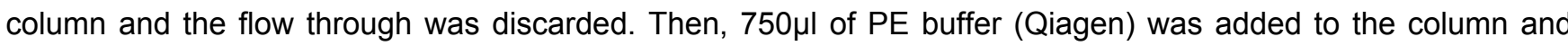
centrifuged at $9,391 \mathrm{~g}$ for $30 \mathrm{~s}$, the flow through discarded, and the empty column centrifuged at 9,391g for $1 \mathrm{~min}$ to remove residual PE buffer. The column was placed in a clean tube and the DNA is eluted by adding $50 \mu$ of EB buffer (10mM Tris-Cl, pH 8.5) followed by centrifugation at $9,391 \mathrm{~g}$ for $1 \mathrm{~min}$.

\section{Short in vivo ssDNA sequencing using DNA adapters}

Step 1-Dephosphorylation and heat denaturation - $1 \mu \mathrm{l}$ of FastAP (1U) was added to the $42-\mu$ l reaction mixture $(20 \mu \mathrm{l}$ DDW, $8 \mu \mathrm{l}$ CircLigase buffer II (10X), $4 \mu \mathrm{l}$ of $\mathrm{MnCl} 2(50 \mathrm{mM})$ and $10 \mu \mathrm{l}$ of the 72 -nt PAGE purified in vivo ssDNA). The reaction was incubated in a thermal cycler with a heated lid for $10 \mathrm{~min}$ at $37^{\circ} \mathrm{C}$, and then at $95^{\circ} \mathrm{C}$ for $2 \mathrm{~min}$. Tubes were put in an ice-water bath for $1 \mathrm{~min}$. 


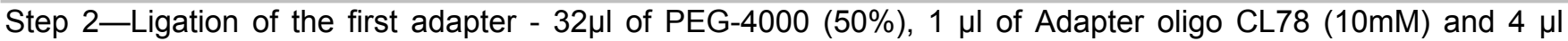
CircLigase II $(100 \mathrm{U} \mu \mathrm{l})$ were added to the resulting solution from step 1 to obtain a final reaction volume of $80 \mu \mathrm{l}$. Then, the reaction mixtures were incubated in a thermal cycler with a heated lid for $1 \mathrm{~h}$ at $60^{\circ} \mathrm{C}$, and $2 \mu \mathrm{l}$ of stop solution $\left(98 \mu \mathrm{l}\right.$ of $0.5 \mathrm{MEDTA}\left(\mathrm{pH}^{1} / 48.0\right)$ and $2 \mu \mathrm{l}$ of Tween-20) were added.

Step 3 -Immobilization of ligation products on beads - beads were prepared by transferring $20 \mu$ from the bead stock solution (MyOne $\mathrm{C} 1$ ) into a 1.5- $\mu$ l tube. The beads were the pelleted using a magnetic rack, and washed twice with $500 \mu \mathrm{l}$ of bead-binding buffer $(7.63 \mu \mathrm{l}$ of DDW, $2 \mu \mathrm{l}$ of $5 \mathrm{M} \mathrm{NaCl}, 100 \mu \mathrm{l}$ of $1 \mathrm{M} \mathrm{Tris-HCl}(\mathrm{pH} 8.0), 20 \mu \mathrm{l}$ of $0.5 \mathrm{M}$ EDTA ( $\mathrm{pH}$ 8.0), $5 \mu \mathrm{l}$ of Tween-20 and $250 \mu \mathrm{l}$ of $20 \%$ (wt/vol) SDS). After wash, beads were re-suspended in

$250 \mu \mathrm{l}$ of bead-binding buffer. In parallel, the ligation reaction from step 2 was incubated for $1 \mathrm{~min}$ at $95 \mathrm{C}$ in a thermal cycler with a heated lid, and then transferred into an ice-water bath for $1 \mathrm{~min}$. Finally, the ligation reaction was transferred to the bead solution and rotated for $20 \mathrm{~min}$ at room temperature.

Step 4-Primer annealing and extension - Beads from step 3 were pelleted using a magnetic rack and supernatant was discarded. Beads were washed once with $200 \mu \mathrm{l}$ of wash buffer $\mathrm{A}(47.125 \mu \mathrm{l}$ of water, $1 \mu \mathrm{l}$ of $5 \mathrm{M} \mathrm{NaCl}, 500 \mu \mathrm{l}$ of $1 \mathrm{M}$ Tris- $\mathrm{HCl}(\mathrm{pH} 8.0), 100 \mu \mathrm{l}$ of $0.5 \mathrm{M}$ EDTA (pH 8.0), $25 \mu \mathrm{l}$ of Tween-20 and $1.25 \mu \mathrm{l}$ of $20 \%$ (wt/vol) SDS) and once with $200 \mu \mathrm{l}$ of wash buffer B $(8.375 \mu \mathrm{l}$ of water, $1 \mu \mathrm{l}$ of $5 \mathrm{M} \mathrm{NaCl}, 500 \mu$ lof $1 \mathrm{M} \mathrm{Tris}-\mathrm{HCl}(\mathrm{pH} 8.0), 100 \mu \mathrm{l}$ of $0.5 \mathrm{M}$ EDTA (pH 8.0) and $25 \mu \mathrm{l}$ of Tween-20). Then, beads were re-suspended with $47 \mu \mathrm{l}$ of the reaction mixture $(40 \mu \mathrm{l}$ of water, $5 \mu \mathrm{l}$ of isothermal amplification buffer (10X), $0.5 \mu \mathrm{l}$ of dNTP mix and $1 \mu \mathrm{l}$ of the extension primer CL9 (100 $\mathrm{mM})$ ). Samples were incubated in a thermal shaker for $2 \mathrm{~min}$ at $65 \mathrm{C}$ and placed in an ice-water bath for $1 \mathrm{~min}$ Then, immediately transferred to a thermal cycler precooled to $15^{\circ} \mathrm{C}$ followed by the addition of $3 \mu \mathrm{l}$ of Bst 2.0 polymerase $(24 \mathrm{U})$. Reaction mixtures were incubated by increasing the temperature by $1 \mathrm{C}$ per min, ramping it up from $15^{\circ} \mathrm{C}$ to $37^{\circ} \mathrm{C}$, while implementing a final incubation step of $5 \mathrm{~min}$ at $37^{\circ} \mathrm{C}$. **all primer sequences are found in Supplementary Table S4.

Step 5-Blunt-end repair - Beads from step 4 were pelleted using a magnetic rack supernatant was discarded. Beads were washed once with $200 \mu$ l of wash buffer A and re-suspended in $100 \mu$ l of stringency wash buffer $(9.5 \mu l$ of water, $250 \mu \mathrm{l}$ of $20 \%$ (wt/vol) SDS and $250 \mu \mathrm{l}$ of $20 \mathrm{X} \mathrm{SSC}$ buffer) and incubated for 3min at $45 \mathrm{C}$ in a thermal shaker. Beads were then pelleted using a magnetic rack and supernatant was discarded. Beads were washed once with $200 \mu \mathrm{l}$ of wash buffer B, Pelleted and wash buffer was discarded. $99 \mu \mathrm{l}$ of the reaction mixture $(86.1 \mu \mathrm{l}$ of water, $10 \mu \mathrm{l}$ of Buffer Tango (10X), $2.5 \mu \mathrm{l}$ of Tween-20 (1\%) and $0.4 \mu \mathrm{l}$ of dNTP mix) was added to the pelleted beads and re-suspended by vortexing. $1 \mu \mathrm{l}$ of T4 DNA polymerase $(5 \mathrm{U})$ was added to the reaction. Reaction mixtures were incubated for $15 \mathrm{~min}$ at $25^{\circ} \mathrm{C}$ in a thermal shaker and the reaction was stopped by adding $10 \mu \mathrm{l}$ of $\operatorname{EDTA}(0.5 \mathrm{M})$.

Step 6- Ligation of second adapter and library elution - Beads from step 5 were pelleted using a magnetic rack supernatant was discarded. Beads were washed with $200 \mu \mathrm{l}$ of wash buffer A, $200 \mu \mathrm{l}$ of stringency wash buffer (with incubation at $45 \mathrm{C}$ for $3 \mathrm{~min}$ ) and $200 \mu \mathrm{l}$ of wash buffer $\mathrm{B}$. Beads were pelleted using a magnetic rack supernatant was discarded. $98 \mu \mathrm{l}$ of the reaction mixture $(73.5 \mu \mathrm{l}$ of water, $10 \mu \mathrm{l}$ of T4 DNA ligase buffer (10X), 10 $\mu \mathrm{l}$ of PEG $4000(50 \%), 2.5 \mu \mathrm{l}$ of Tween-20 (1\%) and $2 \mu \mathrm{l}$ of the double- stranded adapters (100 mM of pre-hybridized oligos CL53 and CL73)) were added together with $2 \mu \mathrm{l}$ of T4 DNA ligase and mixed. Solution was incubated for $1 \mathrm{~h}$ at room temperature.

Step 7-De-immobilization of the DNA from the beads - Beads from step 6 were pelleted using a magnetic rack supernatant was discarded. Beads were washed once with $200 \mu \mathrm{l}$ of wash buffer A and Re-suspended in $100 \mu \mathrm{l}$ of stringency wash buffer, then incubated for $3 \mathrm{~min}$ at $45^{\circ} \mathrm{C}$ in a thermal shaker. Beads were pelleted using a magnetic rack supernatant was discarded. Beads were washed once with $200 \mu \mathrm{l}$ of wash buffer $\mathrm{B}$, then pelleted using a

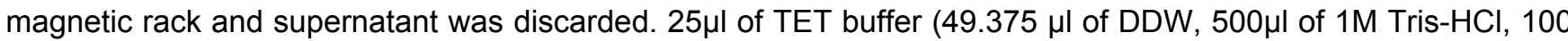
$\mu \mathrm{l}$ of $0.5 \mathrm{M}$ EDTA and $25 \mu \mathrm{l}$ of Tween-20) was added to the pelleted beads and re-suspended by vortexing. Bead 
suspensions were incubated for $1 \mathrm{~min}$ at $95 \mathrm{C}$ in a thermal cycler with a heated lid and Immediately transferred to a magnetic rack. supernatant, which contains the 72-nt in vivo produced ssDNA conjugated to the DNA adapters, was transferred to a fresh tube.

Step 8-PCR amplification and sequencing - The 72-nt in vivo produced ssDNA conjugated to the DNA adapters was PCRed by adding $12 \mu \mathrm{l}$ of the 72 -nt in vivo produced ssDNA conjugated to the DNA adapters (resulting solution from step 7), $28.5 \mu \mathrm{l}$ of water, $5 \mu \mathrm{l}$ of AccuPrim Pfx reaction mix (10X), $2 \mu \mathrm{l}$ of P7L primer, $2 \mu \mathrm{l}$ of P5 primer and 1 $\mu \mathrm{l}$ of AccuPrime Pfx polymerase $(2.5 \mathrm{U} \mu \mathrm{l} /-1)$. The reactions were incubated in a thermal cycler with the following thermal profile; Initial denaturation was carried out at $95 \mathrm{C}$ for $2 \mathrm{~min}$ followed by 27 of PCR cycles, involving denaturation for $15 \mathrm{~s}$ at $95^{\circ} \mathrm{C}$, annealing for $30 \mathrm{~s}$ at $60^{\circ} \mathrm{C}$ and primer extension for $1 \mathrm{~min}$ at $68 \mathrm{C}$. The reaction was terminated by incubating the solution at $72^{\circ} \mathrm{C}$ for $5 \mathrm{~min}$. The resulting PCR solution was then sequenced (Quintarabio) with primer SEQ. Note that oligo P7L was extended in its middle with additional 35-nt (5'-TTGTTTTT CTTTGTTTCTTTTTCTTGTCTTTCTTT-3'). See Table S4 for the complete P7L oligo sequence and all the other oligos used for these assays. This extension had been added to increase the size of the product, thus, allowing the in vivo ssDNA to be fully sequenced. The second oligo used in this assay (P5) was changed to be partially complementary to the in vivo ssDNA and the DNA adapter. A control experiment had been performed in parallel in which the in vivo sSDNA was replaced with the same sequence synthesized by IDT (commercial)

\section{Fluorescence assay for the split YFP assay}

Cells were inoculated in $500 \mu \mathrm{L}$ LB Miller Broth with antibiotics in a 96-well plate covered with a breathable membrane (AeraSeal, Excel Scientific) at $37^{\circ} \mathrm{C}$ at 1,000 r.p.m. (Heidolph Titramax 1000) for $16 \mathrm{~h}$. Overnight cultures are diluted 200 -fold by mixing $2 \mu$ culture into $198 \mu$ l of LB medium containing $10 \mathrm{mM}$ IPTG, $2 \mathrm{mM} \mathrm{L-ara,}$ $0.5 \mathrm{mM} \mathrm{ZnSO} 4,50 \mu \mathrm{g} \mathrm{ml}-1$ spectinomycin, $25 \mu \mathrm{g} \mathrm{ml}-1$ kanamycin and $50 \mu \mathrm{g} \mathrm{ml}-1$ ampicillin. After $8 \mathrm{~h}$ of induction, a $2-\mu \mathrm{l}$ aliquot of culture is prepared for cytometry by diluting it into $198 \mu \mathrm{l}$ of $1 \times$ filtered PBS with $2 \mathrm{mg} \mathrm{ml}-1$ kanamycin.

\section{Cytometry measurements}

Measurements were performed using a Beckman Coulter CytoFLEX S instrument fitted with a 488nm laser for excitation. Emission was measured using the FITC channel at $525140 \mathrm{~nm}$. For each sample, at least 50,000 events were recorded using a $0.6 \mu \mathrm{lls}$ flow rate. Data was gated by forward and side scatter. The fluorescence geometric mean of the total population was calculated. 


\section{List of Figures}

Figure S1. r_oligo Plasmid maps used for all experiments.
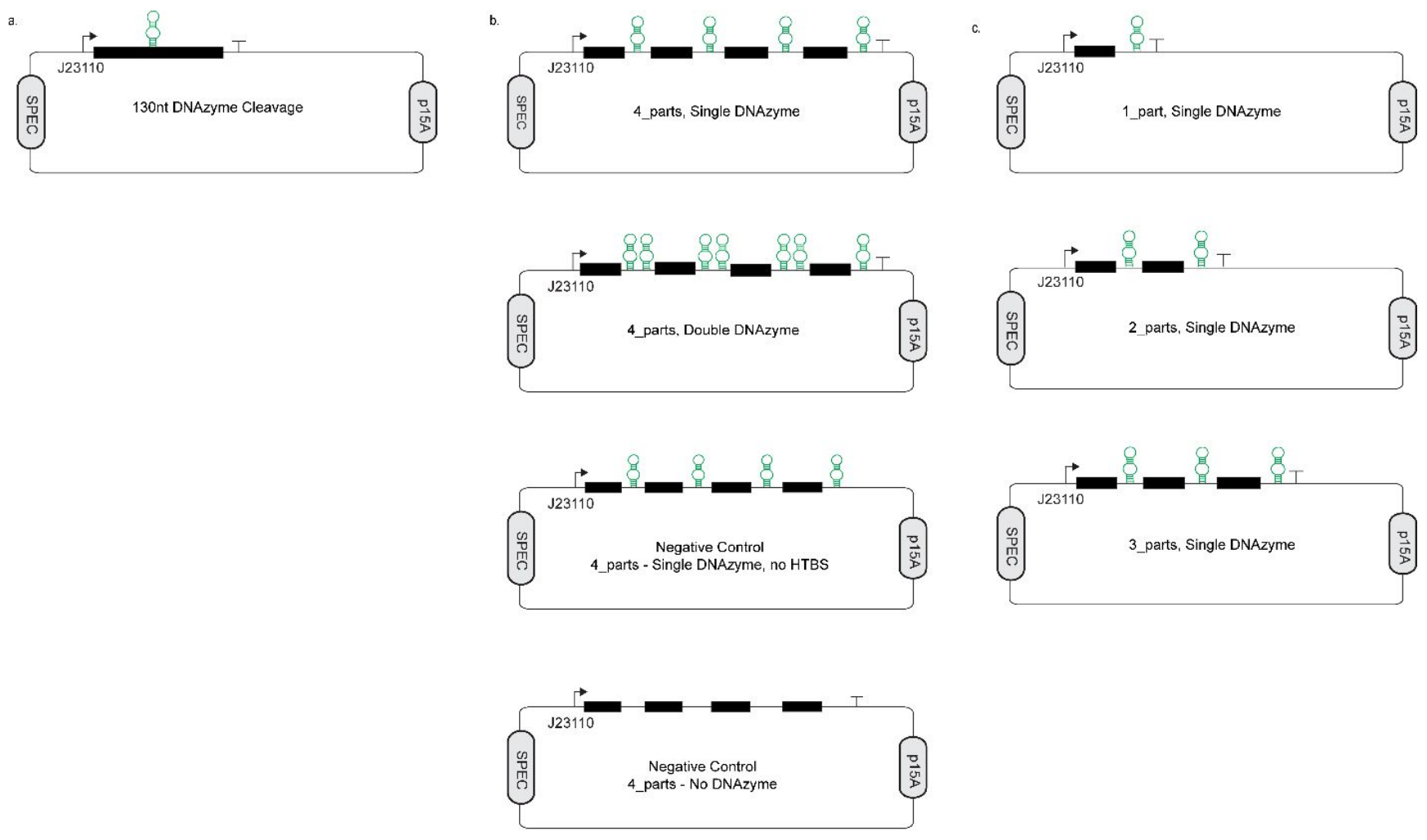

Plasmid maps used in the experiments shown in Figure 2. (b) Plasmid maps used in figure 3, Figure S4 and Figure S6. (c) plasmids used in Figure 3b and Figure S5. Parts beginning with "B" and promotor J23102 are from the Registry of Standard Biological Parts. All plasmids parts and sequences are provided in Tables S1-S3. 
Figure S2. HIV reverse transcriptase Initiator plasmid map.

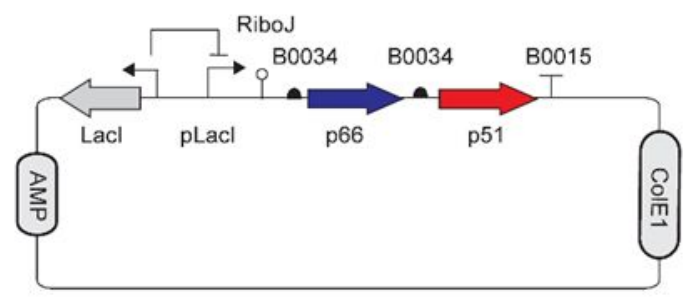

The plasmid was used to generate data shown in all Figures and experiments in the article. Parts beginning with "B" and promotor J23102 are from the Registry of Standard Biological Parts. All sequences are provided in Tables S2 and S3.

Figure S3. Murine Leukemia reverse transcriptase and split YFP sensor Amplifier plasmid maps.

a.

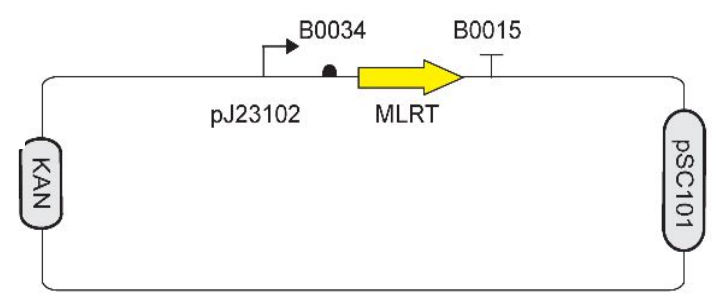

b.
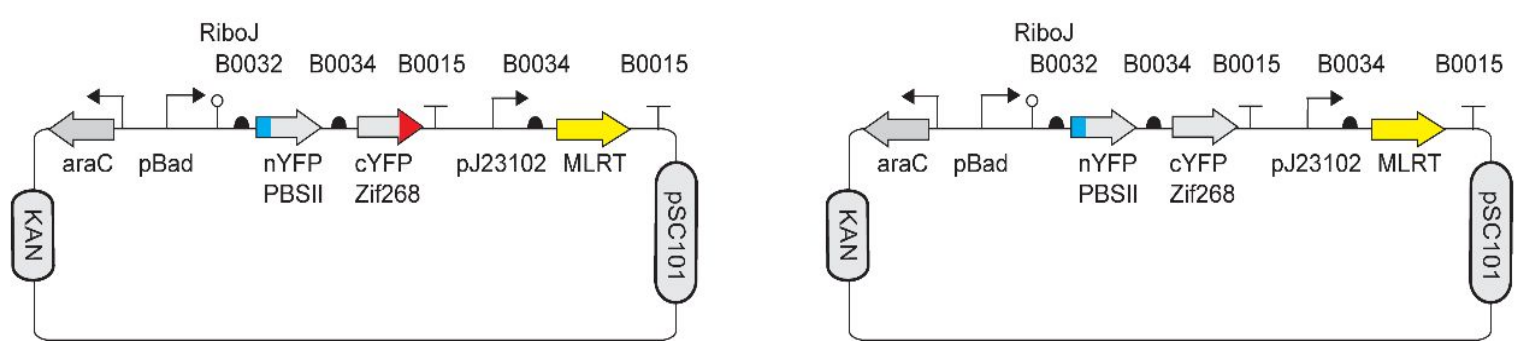

(a) This plasmid was used in the experiment described in Figure 2. (b) The left plasmid was used in experiments described in Figure 3 and Figures S4 and S5. The Right plasmid was used in experiments described in Figure 3d and figure S4. Parts beginning with "B" and promotor J23102 are from the Registry of Standard Biological Parts. All plasmid sequences are provided in supplementary Tables S2 and S3 
Figure S4. Detailed scheme of the 4_part crossover motif and sequence color-coding

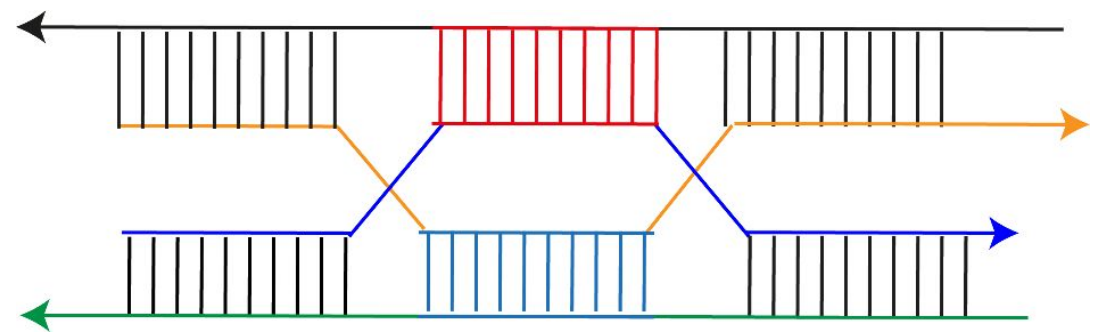

4_parts, single DNAzyme -

TTGC CTCTCAGTTAGTTGAGCTGTATCAGAATGATACGTTGAAGACTGAGAGGAGCATTCGGGAAAGGTGTGACTATGGCTGACGTCAAC TAGTTGAGCTGTCTGCGAAGCAGACGTTGAAGGTTGACGTGGGCGTGCTCACACACCTTTCGGATTGCGCCCCACACATAGTTGAGCTG TATGTGAAACATACGTTGAAGTGTGTGGGCAGCCATAGTAGCGGGTGCGCCGAATGCTCGCCATGCCTAGTTGAGCTGTCGCAGAATGC GACGTTGAAGGGCATGGCTGCGCAATCCCGCACCCGCTTGAGCACGCCCTCC

\section{4_parts, Double DNAzyme -}

TTGCCTCTCAGTTAGTTGAGCTGTATCAGAATGATACGTTGAAGACTGAGAGGAGCATTCGGGAAAGGTGTGACTATGGCTGCGTGGAAC GTTGAAGCGTTACCTGTTAGGTAACGTAGTTGAGCTGTTCCACGTTCGCTCTGCCACTTTAGTTGAGCTGTCGCAGAATGCGACGTTGAA GAAGTGGCAGGGCGTGCTCACACACCTTTCGGATTGCGCACCTTCACGTTGAAGCGTTACCTGTTAGGTAACGTAGTTGAGCTGTGAAG GTTTCGCTCCCCATAATTAGTTGAGCTGTCGCAGAATGCGACGTTGAAGATTATGGGCAGCCATAGTAGCGGGTGCGCCGAATGCTCGA ATGCACGTTGAAGCGTTACCTGTTAGGTAACGTAGTTGAGCTGTGCATTCTTCGCTCGCCATGCCTAGTTGAGCTGTCGCAGAATGCGAC GTTGAAGGGCATGGCTGCGCAATCCCGCACCCGCTTGAGCACGCCCTCC

The scheme presents the arrangement of the oligos within the DNAzyme-Based Operon system compared with the crossover motif DNA scaffold. In the scheme, each oligo is colored and corresponds to the color-coded parts within the DNA sequence. The DNAzyme sequences are color-coded in yellow. Grey sequences in the 4_parts, Double DNAzyme sequence are spacer sequences. 
Figure S5. Expanded schematics and cytometry data corresponding to Figure 3a-c.

Genetic System

ssDNA Expression

DNA Assembly

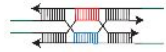

DNA Nanostructure\Protein Complex
Histogram

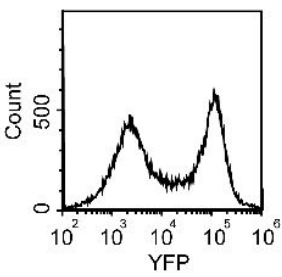

4_parts, Single DNAzyme
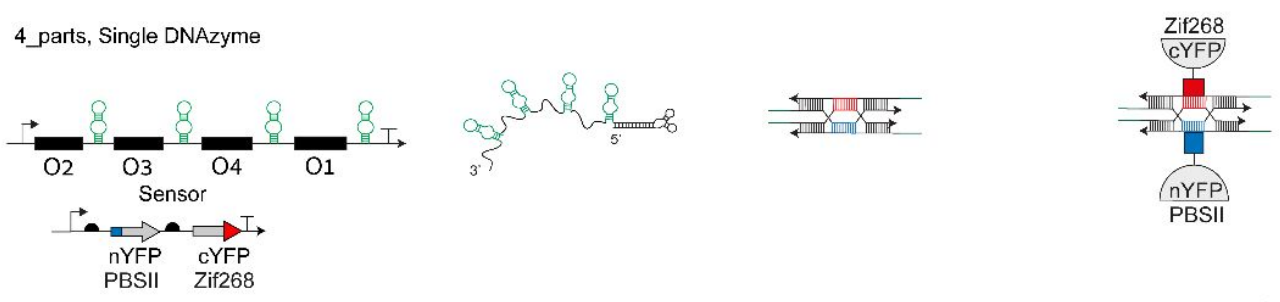

4_parts, Double DNAzyme
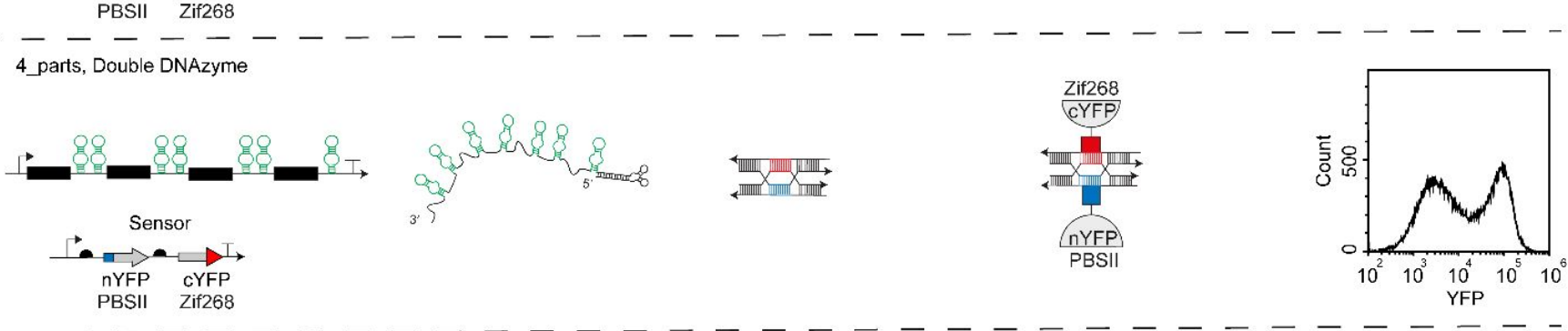

- - - -

4_parts, Single DNAzyme, no HTBS

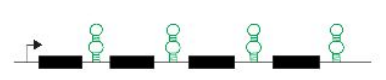

\section{Sensor}

$\underset{n Y F P}{\longrightarrow} \underset{\sim}{\longrightarrow}$, nYFP CYFP PBSII Zif268

BSII

Negative Control

4_parts, No DNAzyme

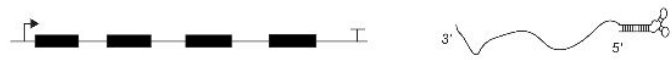

$\underset{\substack{\text { nYFP } \\ \text { PBSII }}}{\stackrel{\text { CYFP }}{\text { Zif268 }}}$
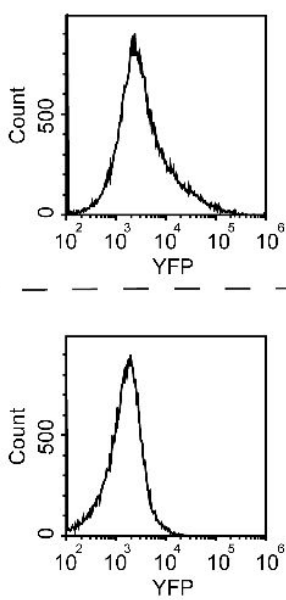

Four different 4_part oligo plasmids have been constructed and used to express the crossover motif and the different controls. All relevant sequences are provided in Supplementary Table S1. All plasmid maps can be found in Supplementary Figures S1b, S2 and S3b. 
Figure S6. Expanded schematics and cytometry data corresponding to Figure 3b.

Genetic System

ssDNA Expression

DNAAssembly

DNA Nanostructure\Protein Complex
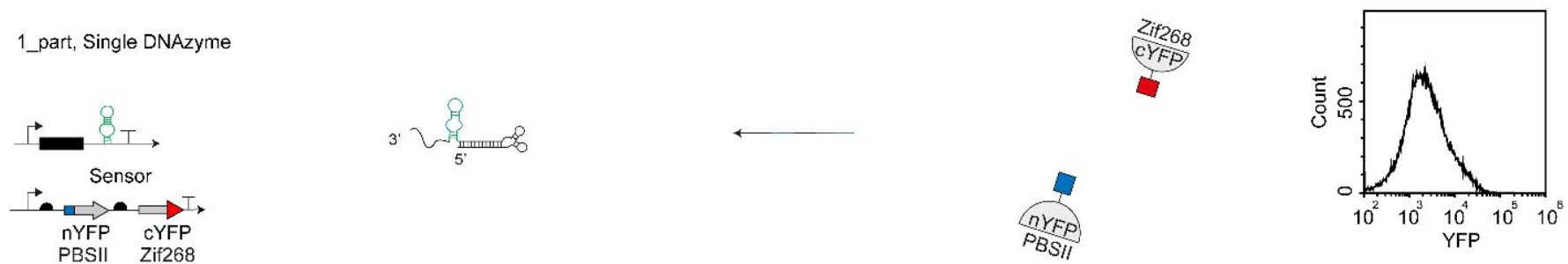

- - - - - -

2_parts, Single DNAzyme
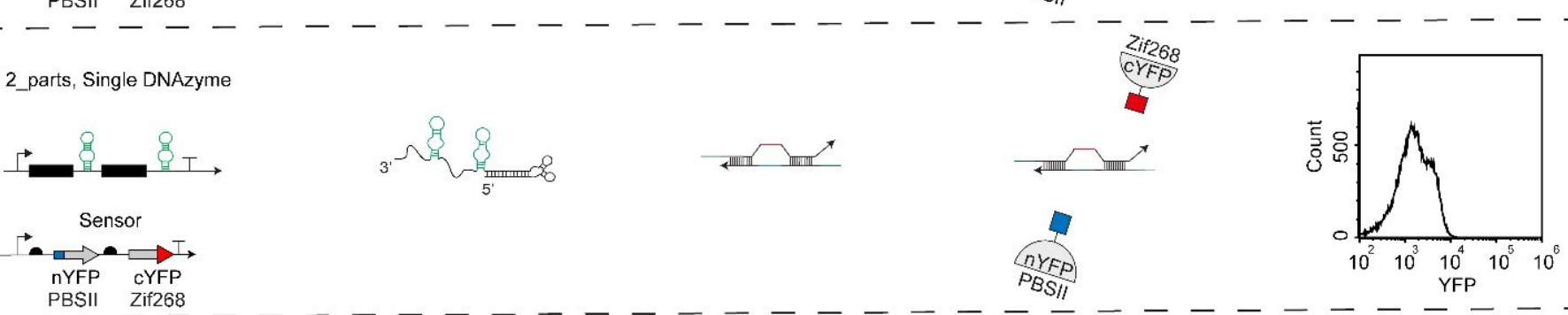

PBSII Zif268

3_parts. Singe DNAzyme
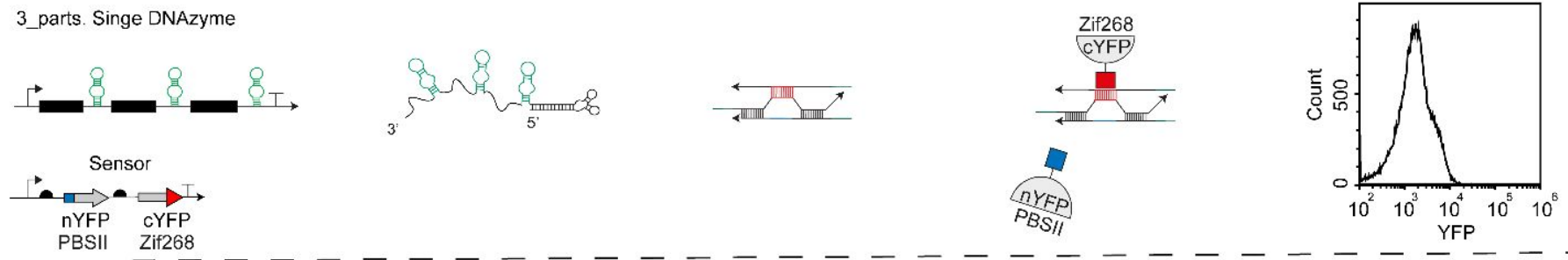

4_parts, Single DNAzyme
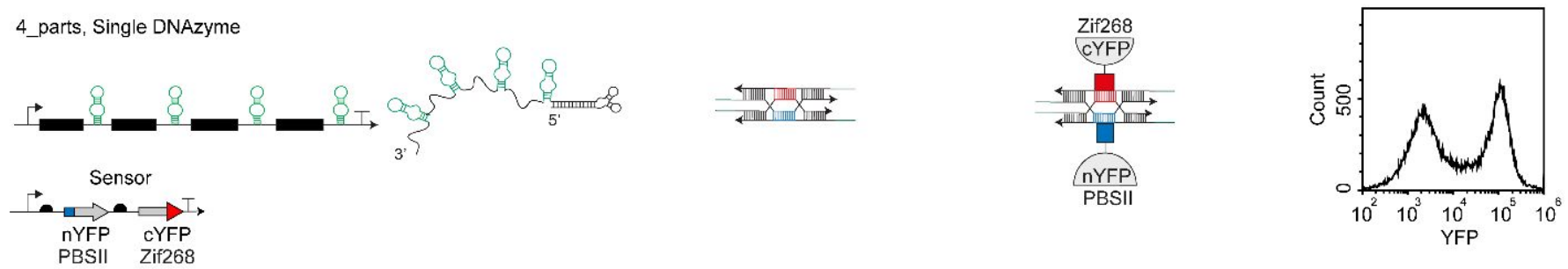

Four different plasmids have been constructed and used to express the different crossover motifs with removed parts shown in Figure 3b. All relevant sequences are provided in Supplementary Table S1. All plasmid maps can be found in Supplementary Figures S1c, S2 and S3b. 
Figure S7. Expanded schematics and cytometry data corresponding to Figure 3d.

Genetic System

4_part, Single DNAzyme

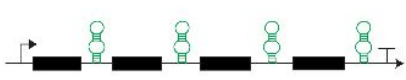

Sensor

$\longrightarrow$.

nYFP CYFP

PBSI

4_part, Double DNAzyme

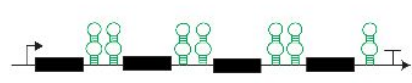

Senso

$\rightarrow \underset{\text { PYFP }}{\Rightarrow} \underset{\text { CYFP }}{\longrightarrow}$ PBSI

Negative Control

4_part - Single DNAzyme, no HTBS

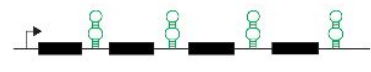

Sensor

$\overrightarrow{\text { nYFP }} \underset{\text { cYFP }}{\vec{P}}$ nYFP

ssDNA Expression

DNAAssembly

DNA Nanostructure Protein Complex

Histogram
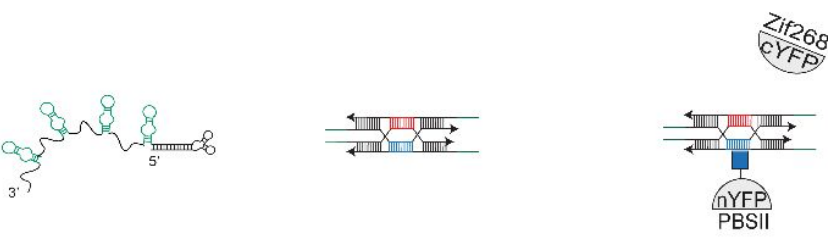

268 YFP
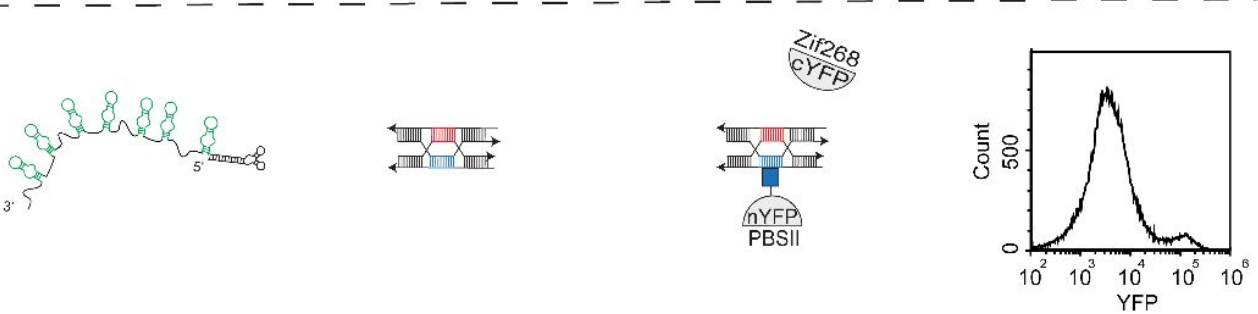

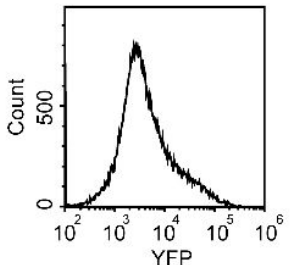

-

Negative Control

4_part - No DNAzyme
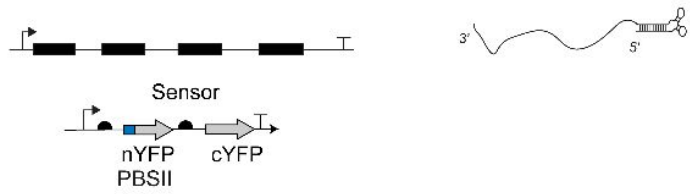

Four different 4-part oligo plasmids have been constructed and used to express the crossover motifs and the different controls. All relevant sequences are provided in Supplementary Table S1. All plasmid maps can be found in Supplementary Figures S1b, S2 and S3b. 
Figure S8. Confocal microscopy images of the DNAzyme based operon system fluorescence in vivo

a.

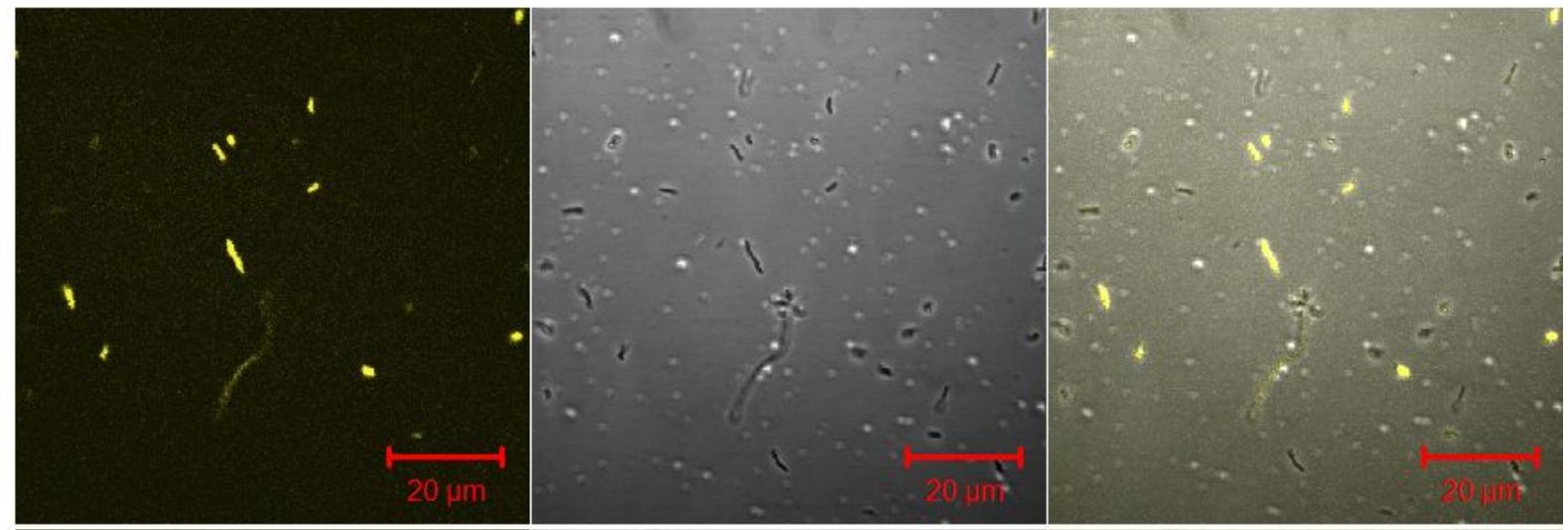

b.

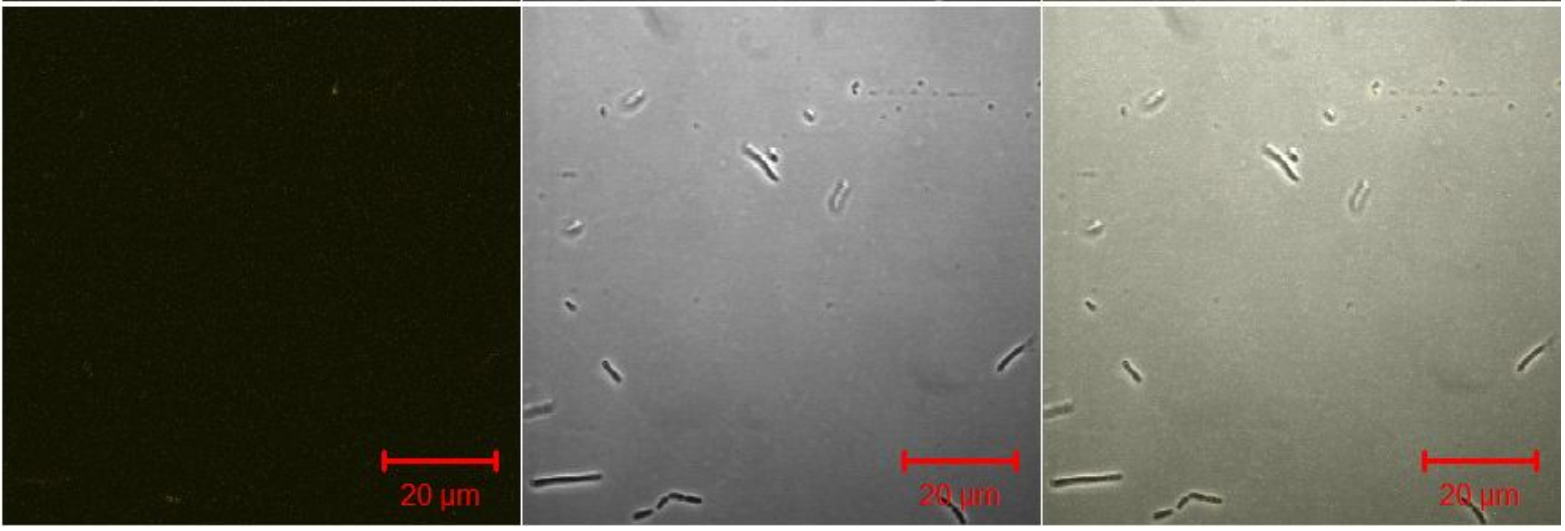

Confocal microscopy images of $E$. coli bacteria expressing the DNAzyme based operon system. Images represent (a) the DNAzyme-based operon system compared with a (b) Negative control experiment as described in figure S5 under " 4_parts, single DNAzyme" and " negative control, 4_parts, no DNAzyme" respectively. Image order from left to right: fluorescence channel, bright field channel and overlay of both channels. All experiments were induced with $10 \mathrm{mM}$ IPTG, $2 \mathrm{mM} \mathrm{L-Ara}$, and $0.5 \mathrm{mM} \mathrm{ZnSO} 4$ and were imaged after 8 hours. All relevant sequences are provided in Supplementary Table 1. All plasmid maps can be found in Supplementary Figures S1b, S2 and S3b. Images were acquired using LSM510 meta Zeiss laser-scanning confocal microscope at X100 with Excitation at $514 \mathrm{~nm}$. Images were further edited using Zeiss LSM image browser. 
Figure S9. In vivo assembly of a 9-part DNA scaffold

a

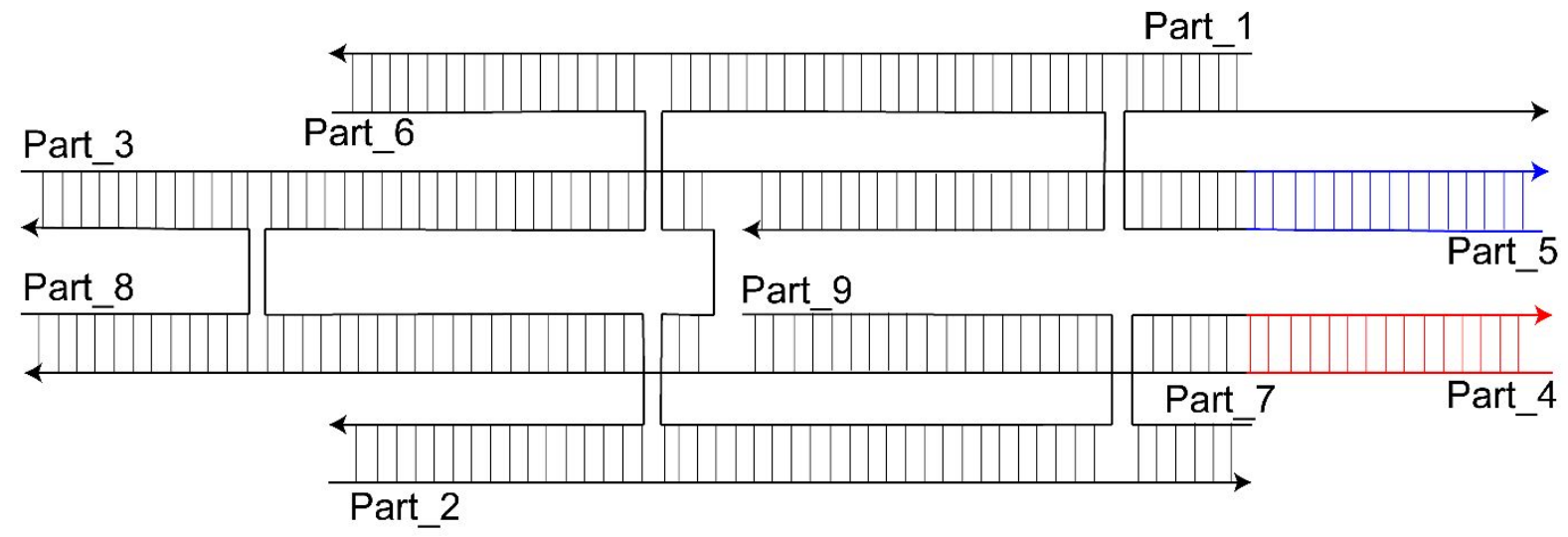

b Fluorescence $\left(\mathrm{au} \times 10^{4}\right)$

4 parts crossover motif, Single DNAzyme

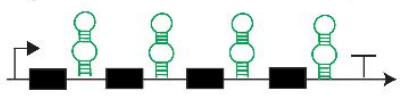

$\begin{array}{llllllll}0 & 2 & 4 & 6 & 8 & 10 & 12 & 14\end{array}$

9 parts scaffold

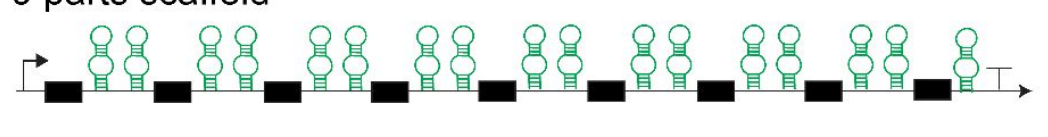

9 parts scaffold, reversed DNAzyme
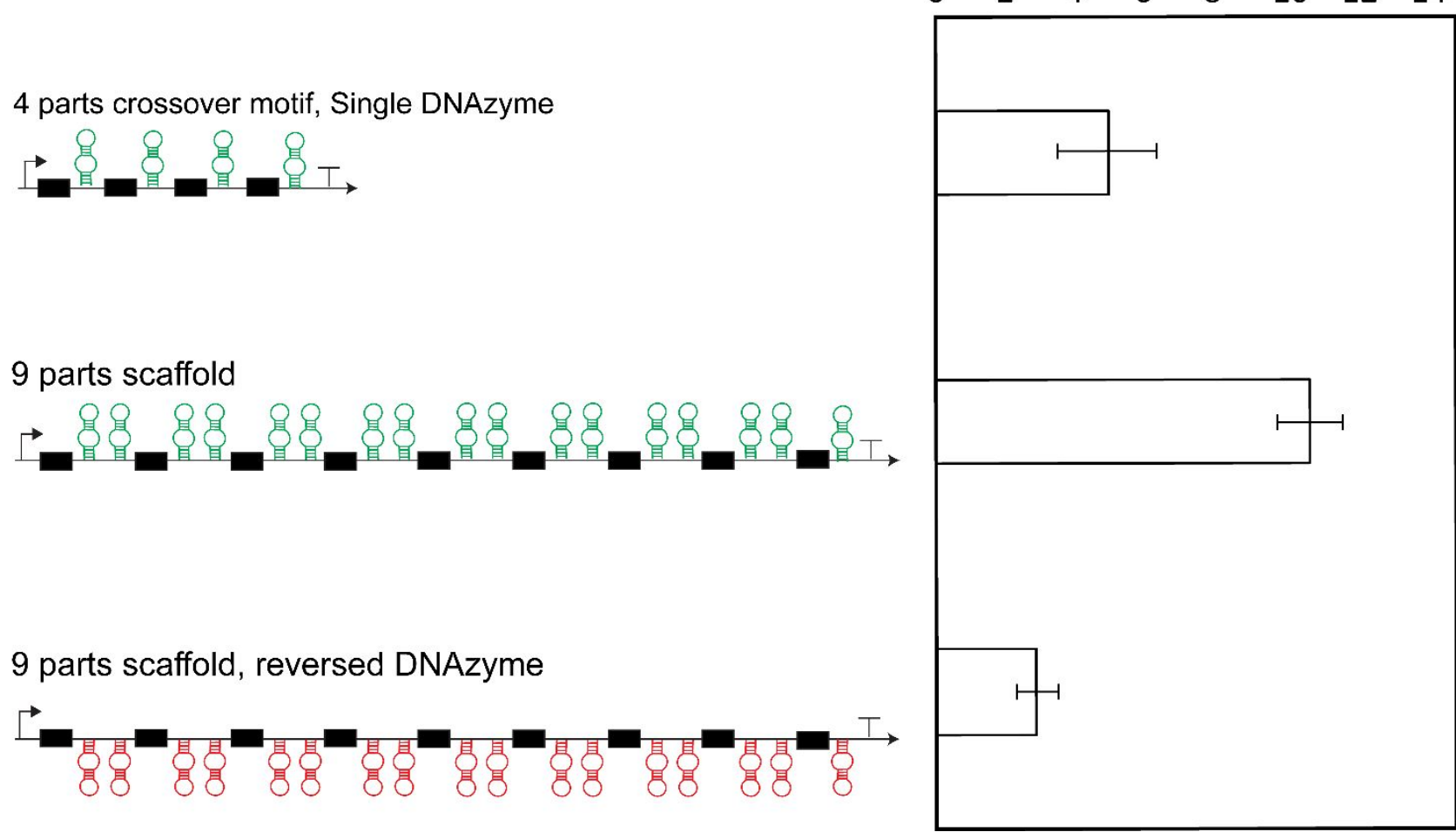

(a) schematic of the 9-part scaffold. Blue and red marks PBS and Zif268 Zn finger binding sites respectively. (b) Fluorescence results of the 9-part scaffold produced by the DNAzyme-based operon systems. A comparison between the 4-part scaffold and the 9-part scaffold. Black boxes represent the parts of each scaffold and are interspaced by DNAzymes (green stem-loops). As a negative control, a reversed DNAzyme-based operon was constructed (red stem-loops). All experiments were induced with $10 \mathrm{mM} \mathrm{IPTG,} 2 \mathrm{mM} \mathrm{L-Ara}$, and $0.5 \mathrm{mM}$ ZnSO4 and were measured after 8 hours. All relevant sequences are provided in Supplementary Table 1. All plasmid maps can be found in Supplementary Figures S1b, S2 and S3b 


\section{List of Tables}

Table S1. ssDNA Sequences.

\begin{tabular}{|c|c|}
\hline Part & Sequence \\
\hline 130bp DNAzyme cleavage & $\begin{array}{l}\text { CGCGGGTTATAGTGCAGTCGTATCATTGCAGACCAAGTAACGTAGTTGAGCTGTCACA } \\
\text { GAATGTGACGTTGAAGCGTTACCTAATTCGGCACATATACACCATGCTCATAAGCAGTC } \\
\text { AAGGAATGGTTTT }\end{array}$ \\
\hline 4_parts, Single DNAzyme & $\begin{array}{l}\text { TTGCCTCTCAGTTAGTTGAGCTGTATCAGAATGATACGTTGAAGACTGAGAGGAGCAT } \\
\text { TCGGGAAAGGTGTGACTATGGCTGACGTCAACTAGTTGAGCTGTCTGCGAAGCAGAC } \\
\text { GTTGAAGGTTGACGTGGGCGTGCTCACACACCTTTCGGATTGCGCCCCACACATAGT } \\
\text { TGAGCTGTATGTGAAACATACGTTGAAGTGTGTGGGCAGCCATAGTAGCGGGTGCGC } \\
\text { CGAATGCTCGCCATGCCTAGTTGAGCTGTCGCAGAATGCGACGTTGAAGGGCATGGC } \\
\text { TGCGCAATCCCGCACCCGCTTGAGCACGCCCTCC }\end{array}$ \\
\hline 4_parts, Double DNAzyme & $\begin{array}{l}\text { TTGCCTCTCAGTTAGTTGAGCTGTATCAGAATGATACGTTGAAGACTGAGAGGAGCAT } \\
\text { TCGGGAAAGGTGTGACTATGGCTGCGTGGAACGTTGAAGCGTTACCTGTTAGGTAAC } \\
\text { GTAGTTGAGCTGTTCCACGTTCGCTCTGCCACTTTAGTTGAGCTGTCGCAGAATGCGA } \\
\text { CGTTGAAGAAGTGGCAGGGCGTGCTCACACACCTTTCGGATTGCGCACCTTCACGTT } \\
\text { GAAGCGTTACCTGTTAGGTAACGTAGTTGAGCTGTGAAGGTTTCGCTCCCCATAATTA } \\
\text { GTTGAGCTGTCGCAGAATGCGACGTTGAAGATTATGGGCAGCCATAGTAGCGGGTGC } \\
\text { GCCGAATGCTCGAATGCACGTTGAAGCGTTACCTGTTAGGTAACGTAGTTGAGCTGT } \\
\text { GCATTCTTCGCTCGCCATGCCTAGTTGAGCTGTCGCAGAATGCGACGTTGAAGGGCA } \\
\text { TGGCTGCGCAATCCCGCACCCGCTTGAGCACGCCCTCC }\end{array}$ \\
\hline 1_part, Single DNAzyme & $\begin{array}{l}\text { TTGCACGTCAACTAGTTGAGCTGTCTGCGAAGCAGACGTTGAAGGTTGACGTGGGCG } \\
\text { TGCTCACACACCTTTCGGATTGCGCCCCA }\end{array}$ \\
\hline 2_part, Single DNAzyme & $\begin{array}{l}\text { TTGCACGTCAACTAGTTGAGCTGTCTGCGAAGCAGACGTTGAAGGTTGACGTGGGCG } \\
\text { TGCTCACACACCTTTCGGATTGCGCCCCACACATAGTTGAGCTGTATGTGAAACATAC } \\
\text { GTTGAAGTGTGTGGGCAGCCATAGTAGCGGGTGCGCCGAATGCTCGCC }\end{array}$ \\
\hline 3_parts, Single DNAzyme & $\begin{array}{l}\text { TTGCCTCTCAGTTAGTTGAGCTGTATCAGAATGATACGTTGAAGACTGAGAGGAGCAT } \\
\text { TCGGGAAAGGTGTGACTATGGCTGACGTCAACTAGTTGAGCTGTCTGCGAAGCAGAC } \\
\text { GTTGAAGGTTGACGTGGGCGTGCTCACACACCTTTCGGATTGCGCCCCACACATAGT } \\
\text { TGAGCTGTATGTGAAACATACGTTGAAGTGTGTGGGCAGCCATAGTAGCGGGTGCGC } \\
\text { CGAATGCTCGCCA }\end{array}$ \\
\hline $\begin{array}{l}\text { Neg. cont. - 4_parts - no } \\
\text { DNAzyme }\end{array}$ & $\begin{array}{l}\text { TTTTTTTTGGCGTGCTCACACACCTTTCGGATTGCGCATTTTTGAGCATTCGGGAAAGG } \\
\text { TGTGACTATGGCTGTTTTTCAGCCATAGTAGCGGGTGCGCCGAATGCTCTTTTTTGCGC } \\
\text { AATCCCGCACCCGCTTGAGCACGCCTTTTT }\end{array}$ \\
\hline $\begin{array}{l}\text { Neg. cont. 4_parts single } \\
\text { DNAzyme - No HTBS }\end{array}$ & $\begin{array}{l}\text { CGCGGGTTATAGTGCAGTCGTATCATTGCAGACCAAGCAACGTAGTTGAGCTGTCACA } \\
\text { GAATGTGACGTTGAAGCGTTGCCTCTCAGTTAGTTGAGCTGTATCAGAATGATACGTTG } \\
\text { AAGACTGAGAGGAGCATTCGGGAAAGGTGTGACTATGGCTGACGTCAACTAGTTGAGC } \\
\text { TGTCTGCGAAGCAGACGTTGAAGGTTGACGTGGGCGTGCTCACACACCTTTCGGATTG } \\
\text { CGCCCCACACATAGTTGAGCTGTATGTGAAACATACGTTGAAGTGTGTGGGCAGCCAT } \\
\text { AGTAGCGGGTGCGCCGATGCTCGCCATGCCTAGTTGAGCTGTCGCAGAATGCGACG } \\
\text { TTGAAGGGCATGGCTGCGCAATCCCGCACCCGCTTGAGCACGCCCTCC }\end{array}$ \\
\hline 9_parts scaffold & $\begin{array}{l}\text { TTGCCTACCTAGGGTCGCCTTGATTCGGCACCTAGTTGAGCTGTGACTAAGAGTCACG } \\
\text { TTGAAGGGTGCCGAGTTCCGGATCACTAATTCCATAGCTTATGCCGGCTTTGCGTAAG } \\
\text { ACCCACAATCGCTTTACTATTCATTAACGTGTGTACGAACGTAACCTGGCAATGGAGAC } \\
\text { GTTGAAGCGGTAAGGTTCCCTTACCGTAGTTGAGCTGTCTCCATGATGCTCTCATATTG } \\
\text { GAAAGTGCCAAAATAGTTGAGCTGTGTAGTTCCTACACGTTGAAGTTTTGGCAGCAGTT } \\
\text { CAGGCCAGCTGATTTTACGTTGAAGCAATTCGTCCGCGATTGTAGTTGAGCTGTAAA } \\
\text { ATCCTACTGGGCGAGATATGCATTCTGAGTTTAGTTGAGCTGTAGATCCAATCTACGTT } \\
\text { GAAGAACTCAGACGACACACCTTTCGTTTACGTTGAAGGTCAACCAGGATGGTTGACT } \\
\text { AGTTGAGCTGTAAACGATTAAGCATAGCAGGCACATCAACCAAAATAGTTGAGCTGTAG } \\
\text { CGGGACGCTACGTTGAAGTTTTGTTCATTGCGGAGTTCAGTCTTAGATGGATCTCGG } \\
\text { ATGCAAGGCCTTCTCTCGTTTTACGTTGAAGACGCCGATAAGATCGGCGTTAGTTGAG } \\
\text { CTGTAAAACGTTAAGCATAGAAGCCAGGCACATCCGCCAAAATAGTTGAGCTGTCTAA } \\
\text { GGATTAGACGTTGAAGTTTTGGCGTGGGCGAACGCTCGGTCGTTTACGTTGAAGCACA } \\
\text { AGTTTCACTTGTGTAGTTGAGCTGTAAACGAGCAAGTTGCGGATACCGTTATTCGAAAA } \\
\text { TAGTTGAGCTGTTAGACCTTCTAACGTTGAAGTTTCGAAAGGTGTGCACGTTAATGAA } \\
\text { TAGTCTCCACTTGCATCCGAGATCCGAACTGCTGCCTTTACGTTGAAGAGGTCCTGAA } \\
\text { GCAGGACCTTAGTTGAGCTGTAAAAGGCATTGCGGAATGGGTCTTATAGTACTGAAAA }\end{array}$ \\
\hline
\end{tabular}




\begin{tabular}{|c|c|}
\hline & $\begin{array}{l}\text { ATAGTTGAGCTGTCATTATGAATGACGTTGAAGTTTTTCAGCTGGCCTATCTAAGACTG } \\
\text { AACTCGCACCGCCGGCATAAGCTATGTCGCCCACGCCTTTACGTTGAAGAGTAAGAGA } \\
\text { AGCTCTTACTTAGTTGAGCTGTAAAGGCGGTGGAGACGGCTGATTACCCTCGAAAATA } \\
\text { GTTGAGCTGTTGCATTGTGCAACGTTGAAGTTTTCGAGAGAAGGCTTGCCAGGTTACG } \\
\text { TTCGTACATCGTCTGAGTTTTTTACGTTGAAGAGCAGAAGTTCCTTCTGCTTAGTTGAG } \\
\text { CTGTAAAAAATTCCATACCACCTGGCGCCAGTCGAAAATAGTTGAGCTGTACCTAACAG } \\
\text { GTACGTTGAAGTTTTCGACCGAGCGTGAATTAGTGATCCGGAACTCGCGCAATGAACC } \\
\text { TTTTCTCC }\end{array}$ \\
\hline $\begin{array}{l}\text { 9_parts scaffold - reversed } \\
\text { DNAzymes }\end{array}$ & $\begin{array}{l}\text { TTGCCTACCTAGGGTCGCCTTGATTCGGCACCTAGTTGAGCTGTGACTAAGAGTCACG } \\
\text { TTGAAGGGTGCCGAGTTCCGGATCACTAATTCCATAGCTTATGCCGGCTTTGCGTAAG } \\
\text { ACCCACAATCGCTTTACTATTCATTAACGGTGTACGAACGTAACCTGGCAATGGAGAC } \\
\text { GTTGAAGCGGTAAGGTTCCCTTACCGTAGTTGAGCTGTCTCCATGATGCTCTCATATTG } \\
\text { GAAAGTGCCAAAATAGTTGAGCTGTGTAGTTCCTACACGTTGAAGTTTTGGCAGCAGTT } \\
\text { CAGGCCAGCTGATTTACGTTGAAGCAATTCGTCCGCGAATTGTAGTTGAGCTGTAAA } \\
\text { ATCCTACTGGGCGAGATATGCATTCTGAGTTTAGTTGAGCTGTAGATCCAATCTACGTT } \\
\text { GAAGAACTCAGACGACACACCTTTCGTTTACGTTGAAGGTCAACCAGGATGGTTGACT } \\
\text { AGTTGAGCTGTAAACGATTAAGCATAGCAGGCACATCAACCAAAATAGTTGAGCTGTAG } \\
\text { CGGGACGCTACGTTGAAGTTTTGGTTCATTGCGGAGTTCAGTCTTAGATGGATCTCGG } \\
\text { ATGCAAGGCCTTCTCTCGTTTTACGTTGAAGACGCCGATAAGATCGGCGTTAGTTGAG } \\
\text { CTGTAAAACGTTAAGCATAGAAGCCAGGCACATCCGCCAAAATAGTTGAGCTGTCTAA } \\
\text { GGATTAGACGTTGAAGTTTTGGCGTGGGCGAACGCTCGGTCGTTTACGTTGAAGCACA } \\
\text { AGTTTCACTTGTGTAGTTGAGCTGTAAACGAGCAAGTTGCGGATACCGTTATTCGAAAA } \\
\text { TAGTTGAGCTGTTAGACCTTCTAACGTTGAAGTTTCGAAAGGTGTGCACGTTAATGAA } \\
\text { TAGTCTCCACTTGCATCCGAGATCCGAACTGCTGCCTTTTACGTTGAAGAGGTCCTGAA } \\
\text { GCAGGACCTTAGTTGAGCTGTAAAAGGCATTGCGGAATGGGTCTTATAGTACTGAAAA } \\
\text { ATAGTTGAGCTGTCATTATGAATGACGTTGAAGTTTTCAGCTGGCCTATCTAAGACTG } \\
\text { AACTCGCACCGCCGGCATAAGCTATGTCGCCCACGCCTTTACGTTGAAGAGTAAGAGA } \\
\text { AGCTCTTACTTAGTTGAGCTGTAAAGGCGGTGGAGACGGCTGATTACCCTCGAAAATA } \\
\text { GTTGAGCTGTTGCATTGTGCAACGTTGAAGTTTTCGAGAGAAGGCTTGCCAGGTTACG } \\
\text { TTCGTACATCGTCTGAGTTTTTACGTTGAAGAGCAGAAGTTCCTTCTGCTTAGTTGAG } \\
\text { CTGTAAAAAATTCCATACCACCTGGCGCCAGTCGAAAATAGTTGAGCTGTACCTAACAG } \\
\text { GTACGTTGAAGTTTTCGACCGAGCGTGAATTAGTGATCCGGAACTCGCGCAATGAACC } \\
\text { TTTTCTCC }\end{array}$ \\
\hline
\end{tabular}

** DNAzymes sequences are bolded 
Table S2. Genetic Part Sequences.

\begin{tabular}{|c|c|}
\hline Part & Sequence \\
\hline pTac & TGTTGACAATTAATCATCGGCTCGTATAATGTGTGGAATTGTGAGCGCTCACAATT \\
\hline $\mathrm{J} 23102$ & TTGACAGCTAGCTCAGTCCTAGGTACTGTGCTAGC \\
\hline $\mathrm{J} 23100$ & TTGACGGCTAGCTCAGTCCTAGGTACAGTGCTAGC \\
\hline HTBS & $\begin{array}{l}\text { AAAAAAAACGTGGCGCCCGAACAGGGACGGATAGCTCAGTCGGTAGAGCATCAGACTTTT } \\
\text { AATCTGAGGGTCCAGGGTTCAAGTCCCTGTTCGGGCGCCACGTTTTTTTT }\end{array}$ \\
\hline riboJ & CTGTCACCGGATGTGCTTTCCGGTCTGATGAGTCCGTGAGGACGAAACAG \\
\hline DNAzyme & GTAACGCTTCAACGTCACATTCTGTGACAGCTCAACTACGTTAC \\
\hline BBa - B0015 & $\begin{array}{l}\text { CCAGGCATCAAATAAAACGAAAGGCTCAGTCGAAAGACTGGGCCTTTCGTTTTATCTGTTG } \\
\text { TTTGTCGGTGAACGCTCTCTACTAGAGTCACACTGGCTCACCTTCGGGTGGGCCTTTCTGC } \\
\text { GTTTATA }\end{array}$ \\
\hline Bba_10500 pBad/araC promotor & $\begin{array}{l}\text { TTATGACAACTTGACGGCTACATCATTCACTTTTTCTTCACAACCGGCACGGAACTCGCTCG } \\
\text { GGCTGGCCCCGGTGCATTTTTTAAATACCCGCGAGAAATAGAGTTGATCGTCAAAACCAAC } \\
\text { ATTGCGACCGACGGTGGCGATAGGCATCCGGGTGGTGCTCAAAAGCAGCTTCGCCTGGCT } \\
\text { GATACGTTGGTCCTCGCGCCAGCTTAAGACGCTAATCCCTAACTGCTGGCGGAAAGATGT } \\
\text { GACAGACGCGACGGCGACAAGCAACATGCTGTGCGACGCTGGCGATATCAAAATTCTG } \\
\text { TCTGCCAGGTGATCGCTGATGTACTGACAAGCTCGCGTACCCGATTATCCATCGGTGGAT } \\
\text { GGAGCGACTCGTTAATCGCTTCCATGCGCCGCAGTAACAATTGCTCAAGCAGATTTATCGC } \\
\text { CAGCAGCTCCGAATAGCGCCCTTCCCCTTGCCCGGCGTTAATGATTTGCCCAAACAGGTC } \\
\text { GCTGAAATGCGGCTGGTGCGCTTCATCCGGGCGAAAGAACCCCGTATTGGCAAATATTGA } \\
\text { CGGCCAGTTAAGCCATTCATGCCAGTAGGCGCGCGGACGAAATAAACCCACTGGTGATA } \\
\text { CCATTCGCGAGCTCCGGATGACGACCGTAGTGATGATCTCTCCTGGCGGGACAGCAA } \\
\text { AATATCACCCGGTCGGCAAACAAATTCTCGTCCCTGATTTTTCACCACCCCCTGACCGCGA } \\
\text { ATGGTGAGATTGAGAATATAACCTTTCATTCCCAGCGGTCGGTCGATAAAAAATCGAGATA } \\
\text { ACCGTTGGCCTCAATCGGCGTTAAACCCGCCACCAGATGGGCATTAAACGAGTATCCCGG } \\
\text { CAGCAGGGGATCATTTTGCGCTTCAGCCATACTTTTCATACTCCCGCCATTCAGAGAAGAA } \\
\text { ACCAATTGTCCATATTGCATCAGACATTGCCGTCACTGCGTCTTTTACTGGCTCTTCTCGCT } \\
\text { AACCAAACCGGTAACCCGCTTATTAAAAGCATTCTGTAACAAAGCGGGACCAAAGCCATG } \\
\text { ACAAAAACGCGTAACAAAAGTGTCTATAATCACGGCAGAAAAGTCCACATTGATTATTTGCA } \\
\text { CGGCGTCACACTTTGCTATGCCATAGCATTTTATCCATAAGATTAGCGGATCCTACCTGAC } \\
\text { GCTTTTTATCGCAACTCTCTACTGTTTCTCCATACCCGTTTTTTTGGGCTAGC }\end{array}$ \\
\hline Lacl & $\begin{array}{l}\text { TCACTGCCCGCTTTCCAGTCGGGAAACCTGTCGTGCCAGCTGCATTAATGAATCGGCCAAC } \\
\text { GCGCGGGGAGAGGCGGTTTGCGTATTGGGCGCCAGGGTGGTTTTTCTTTTCACCAGTGAG } \\
\text { ACTGGCAACAGCTGATTGCCTTCACCGCCTGGCCTGAGAGAGTTGCAGCAAGCGGTC } \\
\text { ACGCTGGTTTCCCCAGCAGGCGAAATCCTGTTTGATGGTGGTAACGGCGGGTATAA } \\
\text { CATGAGCTATCTTCGGTATCGTCGTATCCCACTACCGAGATATCCGCACCAACGCGCAGCC } \\
\text { CGGACTCGGTAATGGCGCGCATTGCGCCCAGCGCCATCTGATCGTTGGCAACCAGCATCG } \\
\text { CAGTGGGAACGATGCCCTCATTCAGCATTTGCATGGTTTGTTGAAACCGGACATGGCACT } \\
\text { CCAGTCGCCTTCCCGTTCCGCTATCGGCTGAATTTATTGCGATGAGATATTATGCCAG } \\
\text { CCAGCCAGACGCAGACGCGCCGAGAAGACTTAATGGGCCGCTAACAGCGCGATTTCC } \\
\text { TGGTGACCCAATGCGACCAGATGCTCCACGCCCAGTCGCGTACCGTCCTCATGGGAGAAA } \\
\text { ATAATACTGTTGATGGGTGTCTGGTCAGAGACATCAAGAAATAACGCCGGAACATTAGTGC } \\
\text { AGGCAGCTTCCACAGCAATGGCATCCTGGTCATCCAGCGGATAGTTAATGATCAGCCCACT } \\
\text { GACGCGTTGCGCGAGAAGATTGTGCACCGCCGCTTTACAGGCTTCGACGCCGCTTCGTTC } \\
\text { TACCATCGACACCACCACGCTGGCACCCAGTTGATCGGCGCGAGATTAATCGCCGCGC } \\
\text { AATTTGCGACGGCGCGTGCAGGGCCAGACTGGAGGTGGCAACGCCAATCAGCAACGACT } \\
\text { GTTTGCCCGCCAGTTGTTGTGCCACGCGGTTGGGAATGTAATTCAGCTCCGCCATCGCCG } \\
\text { CTTCCACTTTTTCCCGCGTTTTCGCAGAAACGTGGCTGGCCTGGTTCACCACGCGGGAAAC } \\
\text { GGTCTGATAAGAGACACCGGCATACTCTGCGACATCGTATAACGTTACTGGTTTCAT }\end{array}$ \\
\hline nYFP - PBSII & $\begin{array}{l}\text { ATGTATCATCACCATCACCATCACACTAGACCGGGTGAAAAACCGTATGCATGTCCTGAAT } \\
\text { GTGGTAAAAGCTTTAGCCAGCGTGCAAATCTGCGTGCACATCAGCGTACCCATACAGGTGA } \\
\text { AAAGCCTTATAAATGCCCAGAATGCGGCAAAGCTTTCACGTAGCGATCATCTGACCACC } \\
\text { CATCAGCGCACACATACTGGCGAGAACCTTACAAATGTCCAGAGGTGGTAAATCATTTA } \\
\text { GCCGTAGTGATGTTCTGGTTCGTCATCAGAGAACCCACACGGGTGGTGGTAGCGGTGGTG } \\
\text { GTTCAGGTGGTAGTACTAGAGTGAGCAAGGGCGAGGAGCTGTTCACCGGGGTGGTGCCC } \\
\text { ATCCTGGTCGAGCTGGACGGCGACGTAAACGGCCACAAGTTCAGCGTGTCCGGCGAGGG } \\
\text { CGAGGGCGATGCACCTACGGCAAGCTGACCTGAAGTCATCTGCACCACCGGCAAGCT } \\
\text { GCCCGTGCCCTGGCCCACCCTCGTGACCACCTTCGGCTACGGCCTGATGTGCTTCGCCCG } \\
\text { CTACCCCGACCACATGAAGCAGCACGACTTCTTCAAGTCCGCCATGCCCGAAGGCTACGT } \\
\text { CCAGGAGCGCACCATCTTCTTCAAGGACGACGGCAACTACAAGACCCGCGCCGAGGTGAA } \\
\text { GTTCGAGGGCACACCCTGGTGAACCGCATCGAGCTGAAGGGCATCGACTTCAAGGAGG } \\
\text { ACGGCAACATCCTGGGGCACAAGCTGGAGTACAACTACAACAGCCACAACGTCTATATCAT } \\
\text { GGCCGACAAGCAGAAGAACGGCATCAAGGTGAACTTCAAGATCCGCCACAACATCTAAA }\end{array}$ \\
\hline
\end{tabular}




\begin{tabular}{|c|c|}
\hline cYFP - Zif268 & $\begin{array}{l}\text { ATGGCCGACAAGCAGAAGAACGGCATCAAGGTGAACTTCAAGATCCGCCACAACATCGAG } \\
\text { GACGGCAGCGTGCAGCTCGCCGACCACTACCAGCAGAACACCCCCATCGGCGACGGCCC } \\
\text { CGTGCTGCTGCCCGACAACCACTACCTGAGCTACCAGTCCGCCCTGAGCAAAGACCCCAA } \\
\text { CGAGAAGCGCGATCACATGGTCCTGCTGGAGTTCGTGACCGCCGCCGGGATCACTCTCG } \\
\text { GCATGGACGAGCTGTACAAGGGTGGCTCTGGCGGTGGGATGGGGGAGCACTAGACCG } \\
\text { GGTGAAAAACCGTATGCATGTCCGGTTGAAAGCTGTGATCGTCGTTTTAGCCGTAGTGATG } \\
\text { AACTGACCCGTCATATTCGTATTCATACAGGTCAGAAACCGTTTCAGTGTCGTATTTGCATG } \\
\text { CGTAATTTTAGCCGTTCAGATCATCTGACCACCCATATTCGTACCCATACTGGCGAAAAACC } \\
\text { GTTTGCCTGTGATATTTGTGGTCGTAAATTTGCACGTTCCGATGAACGTAAACGCCATACCA } \\
\text { AAATTCATACGGGTGGTGGTAGCGGTGGTGGTTCAGGTGGTAGTACTAGACACCACCACC } \\
\text { ACCACCACTAA }\end{array}$ \\
\hline cYFP - wlo Zif268 & $\begin{array}{l}\text { ATGGCCGACAAGCAGAAGAACGGCATCAAGGTGAACTTCAAGATCCGCCACAACATCGAG } \\
\text { GACGGCAGCGTGCAGCTCGCCGACCACTACCAGCAGAACACCCCCATCGGCGACGGCCC } \\
\text { CGTGCTGCTGCCCGACAACCACTACCTGAGCTACCAGTCCGCCCTGAGCAAAGACCCCAA } \\
\text { CGAGAAGCGCGATCACATGGTCCTGCTGGAGTTCGTGACCGCCGCCGGGATCACTCTCG } \\
\text { GCATGGACGAGCTGTACAAGGGTGGCTCTGGCGGTGGGAGTGGGGGAAGCACTAGATAG } \\
\text { ACACCACCACCACCACCACTAA }\end{array}$ \\
\hline
\end{tabular}


Table S3. Reverse Transcriptases sequences.

\begin{tabular}{|c|c|}
\hline Part & Sequence \\
\hline p51 & $\begin{array}{l}\text { ATGCCGATTAGCCCGATTGAAACCGTTCCGGTTAAACTGAAACCGGGTATGGATGG } \\
\text { TCCGAAAGTTAAACAGTGGCCTCTGACCGAAGAAAAAATCAAAGCACTGGTTGAAA } \\
\text { TCTGCACCGAGATGGAAAAAGAAGGCAAAATTAGCAAAATCGGTCCGGAAAATCCG } \\
\text { TATAATACACCGGTTTTGCCATTAAGAAAAAAATAGCACCAAATGGCGCAAACTG } \\
\text { GTGGATTTTCGTGAACTGAATAAACGCACCAGGATTTTGGGAAGTCAGCTGGG } \\
\text { TATTCCGCATCCGGCAGGTCTGAAACAGAAAAAAACGTTACCGTTCTGGATGTTG } \\
\text { GTGATGCATATTTTAGCGTTCCGCTGGATAAAGATTTCCGTAAATATACCGCATTTA } \\
\text { CCATCCCGAGCATTAATAACGAAACACCGGGTATTCGCTATCAGTATAATGTTCTGC } \\
\text { CGCAGGGTTGGAAAGGTAGTCCGGCAATTTTTCAGTGTAGCATGACCAAAATTCTG } \\
\text { GAACCGTTTCGTAAACAGAATCCGGATATTGTGATCTACCAGTATATGGATGATCTG } \\
\text { TATGTTGGTAGCGATCTGGAAATTGTCAGCATCGTACCAAAATTGAAAACTGCG } \\
\text { TCAGCATCTGCTGCGTTGGGGTTTTACCACACCGGATAAAAACATCAGAAAGAAC } \\
\text { CGCCTTTCTGTGGATGGGTTATGAACTGCATCCGGATAAATGGACCGTTCAGCCG } \\
\text { ATTGTTCTGCCGGAAAAGATAGCTGGACCGTTAATGATATTCAGAAACTGGTGGG } \\
\text { TAAACTGAATTGGGCAAGCCAGATTTATGCCGGTATTAAAGTTCGTCAGCTGTGTAA } \\
\text { ACTGCTGCGTGGCACCAAAGCACTGACCGAAGTTGTTCCGCTGACAGAAGAAGCA } \\
\text { GAACTGGAACTGGCAGAAATCGTGAAATTCTGAAAGAACCGGTTCACGGCGTTTA } \\
\text { TTATGATCCGAGCAAAGATCTGATTGCCGAAATCAGAAACAGGGTCAGGGTCAGT } \\
\text { GGACCTATCAGATTTATCAAGAACCGTTAAAAACCTGAAAACCGGCAAATATGCAC } \\
\text { GTATGAAAGGTGCACATACCAACGATGTTAAACAGCTGACCGAAGCAGTTCAGAAA } \\
\text { ATTGCAACCGAAAGCATTGTGATTTGGGGTAAAACCCCGAAATTCAAACTGCCGAT } \\
\text { TCAGAAAGAAACCTGGGAAGCATGGTGGACCGAATATTGGCAGGCAACCTGGATT } \\
\text { CCGGAATGGGATTTGTTAATACCCCTCCGCTGGTTAAACTGTGGTATCAGCTGGA } \\
\text { AAAAGAACCGATTATTGGTGCCGAACCTTTAA }\end{array}$ \\
\hline P66 & 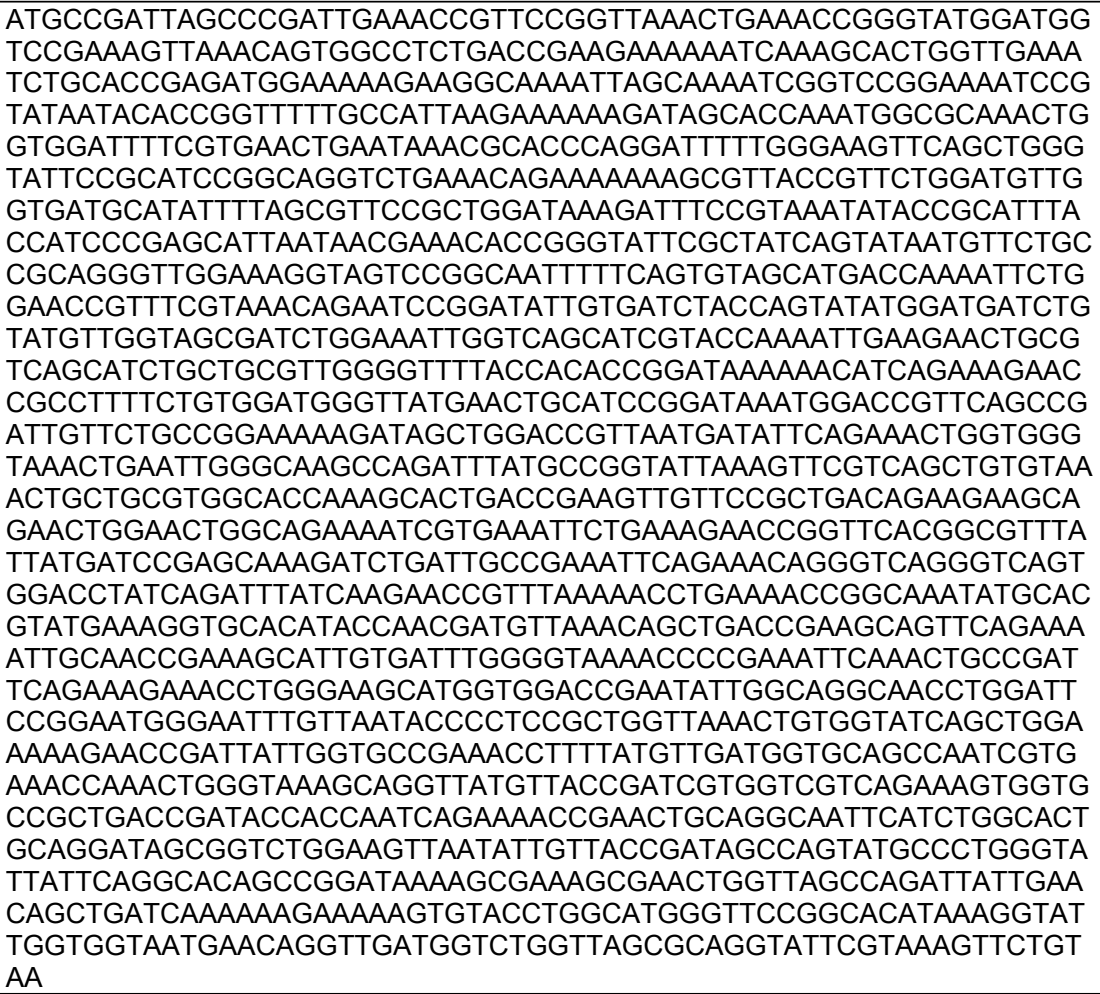 \\
\hline
\end{tabular}


ATGGGTCATAATCATAATCATAATCATAATCATAATCACAACGGTGGAGATGACGAT GACAAGGGTGGTCGACAAGCTTGGATCCCTGCAGGCCTCAGGGCCCGATCGATG GGACCAATGGGGCAGCCCCTGCAAGTGTTGACCCTAAATATAGAAGATGAGTATC GGCTACATGAGACCTCAAAAGAGCCAGATGTTTCTCTAGGGTCCACATGGCTGTCT GATTTTCCTCAGGCCTGGGCGGAAACCGGGGGCATGGGACTGGCAGTTCGCCAA GCTCCTCTGATCATACCTCTGAAAGCAACCTCTACCCCCGTGTCCATAAAACAATAC CCCATGTCACAAGAAGCCAGACTGGGGATCAAGCCCCACATACAGAGACTGTTGG ACCAGGGAATACTGGTACCCTGCCAGTCCCCCTGGAACACGCCCCTGCTACCCGT TAAGAAACCAGGGACTAATGATTATAGGCCTGTCCAGGATCTGAGAGAAGTCAACA AGCGGGTGGAAGACATCCACCCCACCGTGCCCAACCCTTACAACCTCTTGAGCGG GCTCCCACCGTCCCACCAGTGGTACACTGTGCTTGATTTAAAGGATGCCTTTTTCT GCCTGAGACTCCACCCCACCAGTCAGCCTCTCTTCGCCTTTGAGTGGAGAGATCC AGAGATGGGAATCTCAGGACAATTGACCTGGACCAGACTCCCACAGGGTTTCAAAA ACAGTCCCACCCTGTTTGATGAGGCACTGCACAGAGACCTAGCAGACTTCCGGAT CCAGCACCCAGACTTGATCCTGCTACAGTACGTGGATGACTTACTGCTGGCCGCC ACTTCTGAGCTAGACTGCCAACAAGGTACTCGGGCCCTGTTACAAACCCTAGGGAA CCTCGGGTATCGGGCCTCGGCCAAGAAAGCCCAAATTTGCCAGAAACAGGTCAAG TATCTGGGGTATCTTCTAAAAGAGGGTCAGAGATGGCTGACTGAGGCCAGAAAAGA GACTGTGATGGGGCAGCCTACTCCGAAGACCCCTCGACAACTAAGGGAGTTCCTA GGGACGGCAGGCTTCTGTCGCCTCTGGATCCCTGGGTTTGCAGAAATGGCAGCCC CCTTGTACCCTCTCACCAAAACGGGGACTCTGTTTAATTGGGGCCCAGACCAACAA AAGGCCTATCAAGAAATCAAGCAAGCTCTTCTAACTGCCCCAGCCCTGGGGTTGCC AGATTTGACTAAGCCCTTTGAACTCTTTGTCGACGAGAAGCAGGGCTACGCCAAAG GTGTCCTAACGCAAAAACTGGGACCTTGGCGTCGGCCGGTGGCCTACCTGTCCAA AAAGCTAGACCCAGTAGCAGCTGGGTGGCCCCCTTGCCTACGGATGGTAGCAGCC ATTGCCGTACTGACAAAGGATGCAGGCAAGCTAACCATGGGACAGCCACTAGTCAT TCTGGCCCCCCATGCAGTAGAGGCACTAGTCAAACAACCCCCCGACCGCTGGCTT TCCAACGCCCGGATGACTCACTATCAGGCCTTGCTTTTGGACACGGACCGGGTCC AGTTCGGACCGGTGGTAGCCCTGAACCCGGCTACGCTGCTCCCACTGCCTGAGGA AGGGCTGCAACACAACTGCCTTGATATCCTGGCCGAAGCCCACGGAACCCGACCC GACCTAACGGACCAGCCGCTCCCAGACGCCGACCACACCTGGTACACGGATGGAA GCAGTCTCTTACAAGAGGGACAGCGTAAGGCGGGAGCTGCGGTGACCACCGAGA CCGAGGTAATCTGGGCTAAAGCCCTGCCAGCCGGGACATCCGCTCAGCGGGCTG AACTGATAGCACTCACCCAGGCCCTAAAGATGGCAGAAGGTAAGAAGCTAAATGTT TATACTGATAGCCGTTATGCTTTTGCTACTGCCCATATCCATGGAGAAATATACAGA AGGCGTGGGTTGCTCACATCAGAAGGCAAAGAGATCAAAAATAAAGACGAGATCTT TAAATGA 
Table S4. Oligo sequences used during the short ssDNA sequencing assay.

\begin{tabular}{|l|l|}
\hline Name & Sequence \\
\hline CL53 & $\begin{array}{l}\text { CGACGCTCTTC-ddC (ddC }=\text { dideoxycytidine) } \\
\text { CL73 }\end{array}$ \\
\hline CL78 & $\begin{array}{l}\text { [Phosphate]GGAAGAGCGTCGTGTAGGGAAAGAG* } T^{*} G^{*} T^{*} A{ }^{*}=\text { phosphothioate } \\
\text { spacer) } \\
\text { CAAGCAGAAGACGGCATACGAGATCCTGCGATTGTTTTTCTTTGTTTCTTTTTCT } \\
\text { TGTCTTTCTTTG }\end{array}$ \\
\hline P7L & ATGTGACTGGAGTTCAGACGTGT \\
\hline P5 & ACGCTCTTCCCGCGGGTTAT \\
\hline SEQ & CAAGCAGAAGACGGCATACGAGATCCTGCGA \\
\hline
\end{tabular}

\section{References}

1. Engler, C., Gruetzner, R., Kandzia, R. \& Marillonnet, S. Golden Gate Shuffling: A One-Pot DNA Shuffling Method Based on Type IIs Restriction Enzymes. PLoS One 4, e5553 (2009).

2. Gibson, D. G. Enzymatic assembly of overlapping DNA fragments. Methods Enzymol. 498, 349$61(2011)$. 